\title{
Bounded Confidence Evolution of Opinions and Actions in Social Networks
}

\author{
Min Zhan ${ }^{(}$, Gang Kou, Yucheng Dong ${ }^{\circledR}$, Senior Member, IEEE, Francisco Chiclana ${ }^{\circledR}$, Senior Member, IEEE, \\ and Enrique Herrera-Viedma ${ }^{(}$, Senior Member, IEEE
}

\begin{abstract}
Inspired by the continuous opinion and discrete action (CODA) model, bounded confidence and social networks, the bounded confidence evolution of opinions and actions in social networks is investigated and a social network opinions and actions evolutions (SNOAEs) model is proposed. In the SNOAE model, it is assumed that each agent has a CODA for a certain issue. Agents' opinions are private and invisible, that is, an individual agent only knows its own opinion and cannot obtain other agents' opinions unless there is a social network connection edge that allows their communication; agents' actions are public and visible to all agents and impact other agents' actions. Opinions and actions evolve in a directed social network. In the limitation of the bounded confidence, other agents' actions or agents' opinions noticed or obtained by network communication, respectively, are used by agents to update their opinions. Based on the SNOAE model, the evolution of the opinions and actions with bounded confidence is investigated in social networks both theoretically and experimentally with a detailed simulation analysis. Theoretical research results show that discrete actions can attract agents who trust the discrete action, and make agents to express extreme opinions. Simulation experiments results show that social network connection probability, bounded confidence, and the opinion threshold of action choice parameters have strong impacts on the evolution of opinions and actions. However, the
\end{abstract}

Manuscript received May 8, 2020; revised September 16, 2020; accepted November 24, 2020. This work was supported in part by the National Natural Science Foundation of China under Grant 71991460, Grant 71991465, Grant 71871149, Grant 71910107002, and Grant 71725001; in part by the Research Foundation of Education Bureau of Hunan Province, China, under Grant 20B147; and in part by the Spanish State Research Agency under Project PID2019-103880RB-I00/AEI/10.13039/501100011033. This article was recommended by Associate Editor H. A. Abbass. (Corresponding author: Yucheng Dong.)

Min Zhan is with the Key Laboratory of Hunan Province for New Retail Virtual Reality Technology, Institute of Big Data and Internet Innovation, Hunan University of Technology and Business, Changsha 410205, China (e-mail: zhanmin19881005@126.com).

Gang Kou is with the School of Business Administration, Southwestern University of Finance and Economics, Chengdu 611130, China (e-mail: kougang@swufe.edu.cn).

Yucheng Dong is with the Center for Network Big Data and DecisionMaking, Business School, Sichuan University, Chengdu 610065, China (e-mail: ycdong@scu.edu.cn).

Francisco Chiclana is with the Institute of Artificial Intelligence, De Montfort University, Leicester LE1 9BH, U.K., and also with the Andalusian Research Institute on Data Science and Computational Intelligence, University of Granada, 18071 Granada, Spain (e-mail: chiclana@dmu.ac.uk).

Enrique Herrera-Viedma is with the Andalusian Research Institute in Data Science and Computational Intelligence, University of Granada, 18071 Granada, Spain, and also with the Department of Electrical and Computer Engineering, Faculty of Engineering, King Abdulaziz University, Jeddah 21589, Saudi Arabia (e-mail: viedma@decsai.ugr.es).

Color versions of one or more figures in this article are available at https://doi.org/10.1109/TCYB.2020.3043635.

Digital Object Identifier 10.1109/TCYB.2020.3043635 number of agents in the social network has no obvious influence on the evolution of opinions and actions.

Index Terms-Action, bounded confidence, evolution, opinion, social network.

\section{INTRODUCTION}

$\mathbf{I}$ $\mathrm{N}$ THE real world, people often express their opinions regarding products, items, and issues, which are susceptible to change due to the influence of other people's opinions. Indeed, people communicate their opinions and attempt to influence others' opinions in an active give-and-take process governed by collective buying and strategy decisions [1], [8]; consumers communicate with one another about innovation, share their opinions, and ultimately decide to accept or reject a new product [8], [33]; opinion leaders hope to guide others close to them (neighbors) to an expected opinion to achieve a particular goal [54], [55].

Theoretically, the opinion dynamics discipline has been developed to describe the evolution of opinions among a group of individuals who interact among them [4], [14], [39]. Consensus, polarization, or fragmentation is the main characteristics of groups' opinions in the final stage [16], [24], [41]. Based on how opinions are expressed, the existing opinion dynamics models are roughly classed as a continuous or as a discrete model. Classical continuous opinion dynamics models include the DeGroot model [3], [10]; the FriedkinJohnsen model [19]-[21]; the bounded confidence model (e.g., the Deffuant-Weisbuch model [9], [48] and the Hegselmann-Krause model [24], [26]); and the continuous opinion and discrete action (CODA) model [34], while the Voter model [25], [27], [35]; the MR model [22], [28], [46]; and the Snajzd model [42], [45] are representative discrete opinion dynamics models. Over the decades, opinion dynamics has become a hot topic with much attention received from sociology [2], [23]; physics [4], [6]; computer science [11]-[13]; systems and control engineering [15], [17], [40]; and applications in political elections [23]; advertising and markets [7], [32]; and group decision making [30], [47], [50], [51]. Despite this, the existing opinion dynamics models and their extensions are associated the following limitations.

1) Limitation 1: Most of the existing opinion dynamics models focus on the evolution of the opinions [16], [43], [47] and ignore the evolution of 
agents' actions. However, in some issues or events, opinions and actions of agents will evolve with interactions [17], [29], [34], [41]. Therefore, investigating opinions and actions evolutions is a very important and interesting research topic, which so far has been considered only by a limited number of research studies [17], [32], [34].

2) Limitation 2: The aforementioned studies on opinions and actions evolutions models assume that the opinions are inner and invisible, that is, agents only know their own opinions, they cannot obtain but can notice other agents' opinions, which can be used to update/evolve their own opinions [17], [29], [32], [34]. Actually, in real life, in addition to their own opinions, agents can also obtain other agents' opinions when they are connected via a social network that allows their interaction and communication.

Thus, social networking is crucial for agents to obtain other agents' opinions [5], [18], [44], [49]. However, to the best of our knowledge, this is the first research study that focuses on opinions and actions evolutions with bounded confidence in social networks with opinions updating rules based on other agents' opinions or actions. This research study is carried out as per the following sequence of steps.

1) A directed social network is considered with agents as its nodes, and edges representing the connection relationship among the agents. Each agent has a CODA for a certain issue. Meanwhile, we consider the bounded confidence of agents; that is, agents will interact with each other only if the difference in their opinions is smaller than a given bounded confidence threshold. The opinion of an agent is only influenced by the agents whose opinions differ from her/his own no more than a certain confidence level [9], [24].

2) Inspired by the CODA model [34]; the bounded confidence [9], [24]; and social network analysis [31], [36], [38], a model for opinions and actions evolutions with bounded confidence in social networks is proposed, which is called the social network opinions and actions evolutions (SNOAEs) model.

3) Based on the SNOAE model, the evolutions of the opinions and actions with bounded confidence in social networks are investigated by both theoretical analysis and detailed simulation experiments analysis.

The proposed model can be applied to address certain opinions and actions evolutions problems in the real world. For example, people are often confronted with a limited number of choices under certain circumstances, frequently as few as two (e.g., buy or not buy, support or not support, accept or reject, yes or no, and iOS or Android) but have continuous opinions [32], [34]. The opinions of agents can be influenced not only by their friends' opinions but also by other noticed customers' actions. The research results in this article can provide decision support to companies and government in their understanding of the evolutions of public opinions and actions through the analysis of their social networks interactions on a specific issue.
The remainder of this study is organized as follows. Section II introduces the SNOAE model. Section III includes the theoretical analysis. Section IV focuses on the simulation experiments analysis. Finally, concluding remarks are drawn in Section V.

\section{SOCIAL NETWORK OPINIONS AND ACTIONS EVOLUTIONS MODEL}

In the proposed SNOAE model, it is assumed that each agent has a continuous opinion and a discrete action for a certain issue [34]. Agents' opinions are private and invisible, that is, an agent only knows their own opinion and cannot obtain other agents' opinions unless there is a social network connection edge that allows their communication; agents' actions are public and visible to all agents and impact on other agents' actions [17], [32]; both opinions and actions evolve in a directed social network. Specifically, at each time an agent chooses, randomly, another agent to update his/her opinion. Thus, at a particular time, if an agent has a social network connection edge to his/her chosen agent, then the chosen agent's opinion can be obtained and used by the agent to update his/her opinion with bounded confidence; otherwise, the agent updates his/her opinion with bounded confidence based on the noticed actions of other agents.

Assumptions of the SNOAE Model:

1) Let $G(V, E)$ be a directed social network, where $V=$ $\left\{v_{1}, v_{2}, \ldots, v_{n}\right\}$ denotes the set of $n$ agents and $E=$ $\left(e_{i j}\right)_{n \times n}(i, j \in\{1,2, \ldots, n\}$ and $j \neq i)$ represents presence or absence of a directed edges between agents: $e_{i j}=1$ meaning that there is a directed edge from agent $v_{i}$ to agent $v_{j}(i, j \in\{1,2, \ldots, n\}$ and $j \neq i)$; otherwise, $e_{i j}=0$.

2) Let $O(t)=\left\{o_{1}(t), o_{2}(t), \ldots, o_{n}(t)\right\}$ and $A(t)=\left\{A_{1}(t)\right.$, $\left.A_{2}(t), \ldots, A_{n}(t)\right\}$ be the opinions and actions of the $n$ agents in the social network $G(V, E)$ at time $t$ $(t=0,1,2, \ldots)$, respectively, where $o_{i}(t) \in[0,1]$ and $A_{i}(t) \in\{0,1\}(i=1,2, \ldots, n)$.

3) For each agent $v_{j}(j=1,2, \ldots, n)$, the function relating the continuous opinion and the discrete action of agents is described by [32]

$$
A_{j}(t)=\left\{\begin{array}{l}
0, o_{j}(t) \in\left[0, h_{j}\right) \\
1, o_{j}(t) \in\left[h_{j}, 1\right]
\end{array}\right.
$$

where $h_{j}$ is the opinion threshold of action choice of agent $v_{j}$. The larger of the value of the opinion threshold of action choice $h_{j}$, the more corisk-conservative agent $v_{j}$ is; on the contrary, the agent $v_{j}$ has a higher propensity toward risk. Equation (1) explains that the external discrete action depends on the magnitude of the internal continuous opinion [32], as the schematic diagram in Fig. 1 shows.

For example, when buying a product, $A_{j}(t)=0$ describes the "not buy" action while $A_{j}(t)=1$ describes the "buy" action. Thus, a risk-preference agent may assume an opinion threshold of action choice of $h_{j}=0.3$, which means that he/she is willing to buy the product $\left(A_{j}(t)=1\right)$ when his/her opinion is more than or equal to 0.3 [i.e., $o_{j}(t) \geq 0.3$ ]; otherwise, he/she will not 


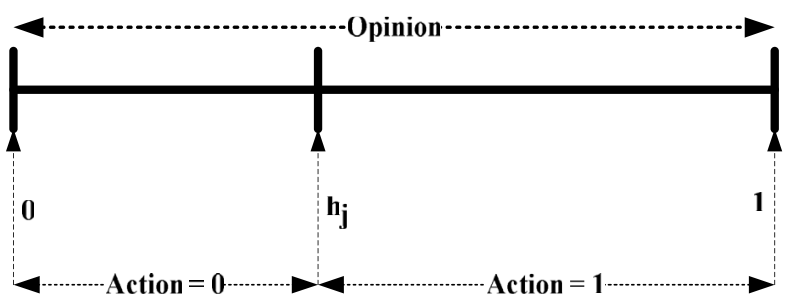

Fig. 1. Schematic of continuous opinions and discrete actions of agents.

TABLE I

Parameters in the SNOAe Model

\begin{tabular}{ll}
\hline \hline PARAMETERS & \multicolumn{1}{c}{ Meaning of Parameters } \\
\hline$o_{j}(t)$ & The opinion of agent $v_{j}$ at a time $t$ \\
$A_{j}(t)$ & The action of agent $v_{j}$ at a time $t$ \\
$h_{j}$ & The opinion threshold of action choice of agent $v_{j}$ \\
$\alpha$ & The convergence parameter of agent $v_{i}$ being \\
& influenced by the agent $v_{j}$ \\
$\varepsilon_{i}$ & The bounded confidence of agents $v_{i}$ \\
$e_{i j}$ & The directed edge from agent $v_{i}$ to agent $v_{j}$ \\
\hline \hline
\end{tabular}

to buy the product; however, a risk-conservative agent may assume an opinion threshold of action choice of $h_{j}=0.7$, and will select the buy action only when his/her opinion verifies $o_{j}(t) \geq 0.7$.

4) Let $\varepsilon_{i}$ be the bounded confidence of agents $v_{i}$. At each time value $t$, it is assumed that each agent $v_{i}(i=1,2, \ldots, n)$ randomly chooses another agent $v_{j}$ $(j \neq i)$ to update his/her opinion. If $e_{i j}=1$, then agent $v_{i}$ can communicate with and obtains the opinion of agent $v_{j}$, which is used to update his/her opinion with bounded confidence $\varepsilon_{i}$. If $e_{i j}=0$, then the agent $v_{i}$ cannot communicate with the agent $v_{j}$ to obtain the opinion of agent $v_{j}$, but he/she can notice the action of the agent $v_{j}$, which is used to update his/her opinion with bounded confidence $\varepsilon_{i}$. Thus, the opinion of agent $v_{i}(i \in\{1,2, \ldots, n\}$, $i \neq j)$ at the time $t+1(t=0,1,2, \ldots)$ evolves as per the following:

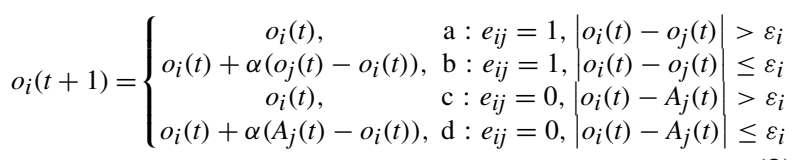

where $\alpha \in(0,0.5]$ is the convergence parameter of the agent $v_{i}$ being influenced by the agent $v_{j}$. The action of agent $v_{i}(i=\{1,2, \ldots, n\})$ at a time $t+1$ $(t=0,1,2, \ldots)$ evolves based on the new opinion $o_{i}(t+1)$ and (1). Table I summarizes the parameters in the SNOAE model.

Comparison Between the SNOAE Model and the DW and the HK Models: Like the DW model [9], [29], [48] and HK model [24], the SNOAE model also relies on the idea of repeated averaging opinions under bounded confidence [16].
In fact, under certain additional assumptions, the SNOAE model extends the DW model. Indeed, when the social network is fully connected and agents meet in random pairwise encounters where they do or do not compromise, then (2) of the SNOAE model coincides with the evolution of opinions equation of the DW model. However, they differ in their evolution of opinions regime as follows.

1) The DW model [9], [48] and the HK model [24] assume that agents always know other agents' opinions at each time and update their opinions based on other agents' opinions under bounded confidence. In the proposed SNOAE model, agents only know the opinions of those agents directly connected with; otherwise, they can only notice other agents' actions. Therefore, in the proposed SNOAE model agents update their opinions with bounded confidence based on the noticed actions of agents they are not connected or the opinions of agents they are directly connected with.

2) The DW model [9], [48] and the HK model [24] only consider opinion evolution, while the SNOAE model considers both opinion and action evolution.

3) The HK model [24] is more suitable for formal meetings in which each agent moves to the average opinion of all agents under bounded confidence; the DW model [9], [48] is suited for agents' random pairwise interactions under bounded confidence within large populations; the SNOAE model can be used in situations where the opinions of people can be influenced not only by his/her friends' opinions but also can be influenced by other nonfriends' actions. For example, regarding the purchase or not of a product, people take into account their friends' opinions on the product but also they are influenced by the noticed purchasing behavior of other customers.

In real life, people are heterogeneous due to their different backgrounds and personalities; which can be formally modeled by the utilization of different parameters to characterize different agents. However, in order to simplify the problem at hand, reasonable assumptions are usually adopted in theoretical studies, which in this article are: all the agents have the same opinion threshold of action choice $h$, bounded confidence $\varepsilon$, and convergence parameter $\alpha$.

\section{Theoretical Analysis of the SNOAE Model}

In this section, the evolution of opinions and actions in the SNOAE model are supported with detailed theoretical study.

Proposition 1: For any chosen agent $v_{j}$ of agent $v_{i}$, if $e_{i j}=0$, $A_{j}(t)=0$ and $\left|o_{i}(t)-A_{j}(t)\right| \leq \varepsilon(i \neq j)$ at each time $t$ $(t=0,1,2, \ldots)$, then $\lim _{t \rightarrow \infty} o_{i}(t)=0$.

Proof: The proof of Proposition 1 is provided in the Appendix.

Proposition 1 states that if an agent always use the noticed other agents' discrete action of nonsupport to update his/her opinion, and the distance between the agent's opinion and the noticed discrete action of nonsupport is always within the bounded confidence, then the opinion of the agent can 
be completely attracted by the discrete action of nonsupport until the opinion value of the agent is 0 .

Example 1: There are two agents $A$ and $B$. Let their opinion thresholds of action choice are 0.5. In the initial time, the opinion and action of agent $A$ are 0.1 and 0 , the opinion and action of agent $B$ are 0.25 and 0 , respectively. Because there is no connection and communication between $A$ and $B$, they update their opinions depending on the other side action at each time. Let the bounded confidences of agents are 0.15. Because the distance between the action value 0 of agent $B$ and the opinion value of agent $A$ is always less than the bounded confidence value 0.15 , then the opinion value of agent $A$ will gradually near by the action value 0 of agent $B$ in the process of opinion and action evolution, and after a long time iteration the opinion of agent $A$ will be 0 .

Proposition 2: For any chosen agent $v_{j}$ of agent $v_{i}$, if $e_{i j}=0$, $A_{j}(t)=1$ and $\left|o_{i}(t)-A_{j}(t)\right| \leq \varepsilon(i \neq j)$ at each time $t(t=0,1,2, \ldots)$, then $\lim _{t \rightarrow \infty} o_{i}(t)=1$.

Proof: The proof of Proposition 2 is provided in the Appendix.

Proposition 2 states that if an agent always use the noticed other agents' discrete action of support to update his/her opinion, and the distance between the agent's opinion and the noticed discrete action of support is always within the bounded confidence, then the opinion of the agent can be completely attracted by the discrete action of support until the opinion value of the agent is 1 .

Example 2: There are two agents $A$ and $B$. Let their opinion thresholds of action choice are 0.5. In the initial time, the opinion and action of agent $A$ are 0.9 and 1, the opinion and action of agent $B$ are 0.8 and 1, respectively. Because there is no connection and communication between $A$ and $B$, they update their opinions depending on the other side action at each time. Let the bounded confidences of agents are 0.15 . Because the distance between the action value 1 of agent $B$ and the opinion value of agent $A$ is always less than the bounded confidence value 0.15 , then the opinion value of agent $A$ will gradually near by the action value 1 of agent $B$ in the process of opinion and action evolution, and after a long time iteration the opinion value of agent $A$ will be 1 .

Proposition 3: When $p=0, \varepsilon<\min \{h, 1-h\}$, then $A_{i}(t+$ 1) $=A_{i}(t)(i=1,2, \ldots, n, t=0,1,2, \ldots)$.

Proof: The proof of Proposition 3 is provided in the Appendix.

Proposition 3 shows that when network connection probability is 0 , the bounded confidence is less than both the opinion threshold of action choice and 1 minus the opinion threshold of action choice, then the actions of agents do not change, that is, all the actions of agents are stable.

That is to say, when there is no connection edges and communication in a group of agents, agents depend on the noticed other agents' actions to update their opinions. Due to the bounded confidence is less than the opinion threshold of action choice and less than that 1 minus the opinion threshold of action choice, an agents' opinion is only influenced by the noticed other agents' action, which is the same with the agents' action. Their actions keep stability, even though

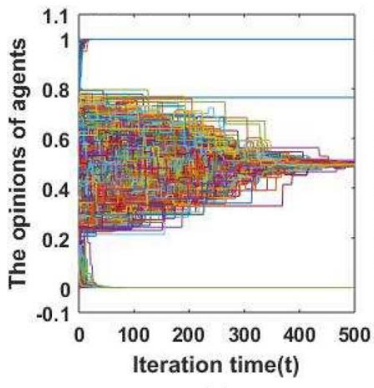

(a)

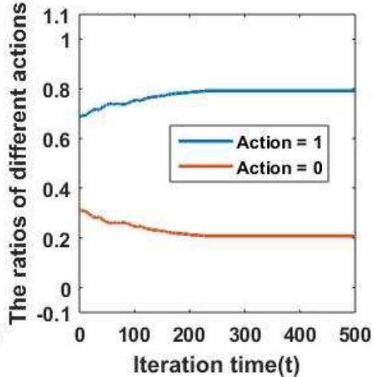

(b)
Fig. 2. Evolutions of (a) opinions and (b) actions for $n=500$ agents over time, and fixed parameter values $p=0.1, \varepsilon=0.2, h=0.3, \alpha=0.5$.

their opinions are still changing. Examples 1 and 2 can help to understand Proposition 3.

Proposition 4 below shows that agents' actions do not change, if there is no edge in social network between agents with action support and agents with action nonsupport and the distances between the opinion of agent with action support (or nonsupport) and the action of agent with action nonsupport (or support) are always larger than the bounded confidence.

Let $V^{0}(t)$ be the set of agents with action nonsupport at time $t$, that is, $A_{i}(t)=0$, where $v_{i} \in V^{0}(t)$; and let $V^{1}(t)$ be the set of agents with action support at time $t$, that is, $A_{j}(t)=1$ where $v_{j} \in V^{1}(t)$.

Proposition 4: In social network $G(V, E)$, for any two agents $v_{i} \in V^{0}(t)$ and $v_{j} \in V^{1}(t)$ at time $t$, if $e_{i j}=0$ and $\left|o_{i}(t)-A_{j}(t)\right|>\varepsilon$, and $e_{j i}=0$ and $\left|o_{j}(t)-A_{i}(t)\right|>\varepsilon$, then $A_{i}(t+1)=A_{i}(t)$ and $A_{j}(t+1)=A_{j}(t)$.

Proof: The proof of Proposition 4 is provided in the Appendix.

Proposition 4 can help us to understand that due to the limitation of bounded confidence and network connection, internal communication between agents with the same action does not change the action of agents.

\section{Simulation Experiments Analysis OF THE SNOAE MODEL}

This section provides simulation experiments analysis of the evolution of opinions and actions in SNOAE model by considering: 1) the final ratio of agents in each action and 2) the total change ratio of agents in each action, with different social network structures (varying the connected probability parameter value), bounded confidence, and opinion threshold of action choice parameter values.

It was found that the evolutions of opinions do not always achieve a stable state after long-time iteration, which is visualized in Fig. 2 for 500 agents in the network with connected probability parameter value of $p=0.1$, a bounded confidence parameter value of $\varepsilon=0.2$, an opinion threshold of action choice parameter value of $h=0.3$, a convergence parameter value of $\alpha=0.5$, and iteration time of $t=500$. The evolution ends after per agent running 500 times [17], [32].

The following notation is used in the simulation experiments: $k \in\{0,1\}$ represents the action value of agents, where 
TABLE II

Parameters Setting in Simulation Experiments in Section IV-A

\begin{tabular}{ll}
\hline \hline PARAMETERS & MEANING OF PARAMETERS \\
\hline$n \in[100,500]$ & The numbers of agents \\
$p \in[0,1]$ & Network connection probabilities \\
$\varepsilon=0.2$ & Bounded confidence \\
$h=0.3$ & Opinion threshold of action choice \\
$\alpha=0.5$ & The convergence parameter \\
$t=0,1,2, \ldots, 500$ & Iteration times \\
\hline \hline
\end{tabular}

$k=0$ be the action of nonsupport and $k=1$ be the action of support. $N_{k}^{t}$ denotes the number of agents with action $k$ at time $t$, the final ratio of agents with action $k$ at time $t$ is $p_{k}^{t}=N_{k}^{t} / n$, and the total change ratio of agents with action $k$ from the initial time to time value $t$ is $\Delta p_{k}^{t}=p_{k}^{t}-p_{k}^{0}$.

\section{A. Impacts of the Social Network Structure on the Evolution of Opinions and Actions}

In real life, especially in a social network framework, agents' opinions are often influenced by other agents' opinions and actions. The presence or absence of social network connections between agents implies that agents can obtain other agents' opinions or can notice other agents' actions, respectively. Previous research studies reveal that social networks have important impacts on opinions evolutions. The impacts of the social network structure, defined by directed ErdôsRényi (ER) random graphs by its numbers of $(n)$ and connected probability parameter value $(p)$, on opinions and actions evolution is of interest to the present study.

Simulation method aims to determine the final ratio of agents with action $k$ at time $t=500$ (i.e., $p_{k}^{500}, k=0$ or $k=1$ ) and the total change ratio of agents with action $k$ the initial time to time value $t=500$ (i.e., $\Delta p_{k}^{500}, k=0$ or 1 ).

Simulation method:

Input: $n, p, h, \alpha$ and $\varepsilon$.

Output: The average $p_{k}^{500}$ and average $\Delta p_{k}^{500}(k=0,1)$.

Step 1: Let $t=0$. Select initial opinions of agents $O(0)=\left\{o_{1}(0), o_{2}(0), \ldots, o_{n}(0)\right\}$ uniformly and randomly from $[0,1]$; apply (1) to obtain the initial actions of agents $A(0)=\left\{A_{1}(0), A_{2}(0), \ldots, A_{n}(0)\right\}$.

Step 2: Generate a directed ER random graph $G(V, E)$ based on $n$ and $p$; the directed edge $e_{i j}$ from agent $v_{i}$ to agent $v_{j}(i \neq j)$ is obtained.

Step 3: Apply (2) to obtain the opinions of agents $o_{i}(t+1)$, and apply (1) to obtain the actions of agents $A_{i}(t+1)$.

Step 4: When $t=500$, obtain $p_{k}^{500}$ and $\Delta p_{k}^{500}(k=0,1)$.

Step 5: After 500 independent realizations, output average $p_{k}^{500}$ and average $\Delta p_{k}^{500}(k=0,1)$.

Table II summarizes the parameter settings implemented in the simulation experiment described above.

In the context of different numbers of agents $n$ and different connected probability values $p$, the evolution results of opinions and actions represented by the average $p_{k}^{500}$ and average $\Delta p_{k}^{500}(k=0,1)$ are shown in Fig. 3.

The following observations are noted.

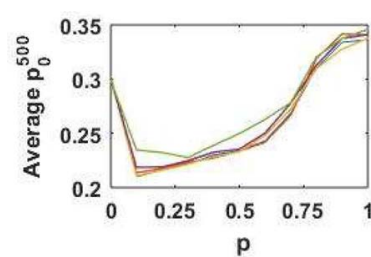

(a)

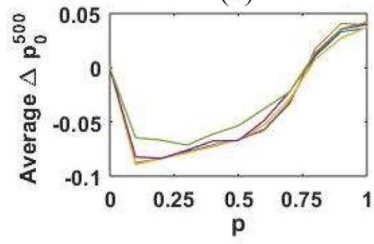

(c)

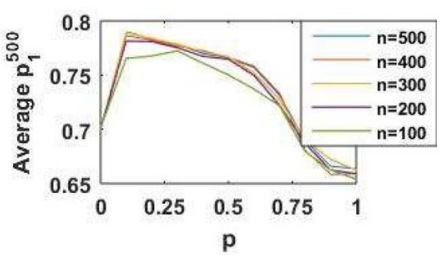

(b)

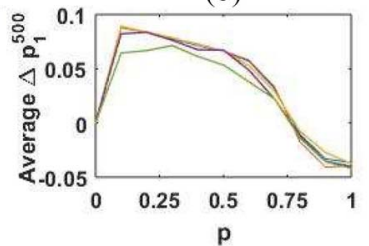

(d)
Fig. 3. Evolution of opinions and actions, represented by average $p_{k}^{500}$ and average $\Delta p_{k}^{500}$ ( $k=0$ or 1) after 500 times independent simulation realizations, for different numbers of agents $n$ with respect to the network connection probability parameter $p$, and fixed parameter values $h=0.3, \alpha=$ $0.5, \varepsilon=0.2$, and $t=500$. (a) The average $p_{k}^{500}(k=0)$. (b) The average $p_{k}^{500}(k=1)$. (c) The average $\Delta p_{k}^{500}(k=0)$. (d) The average $\Delta p_{k}^{500}(k=1)$

1) For a network connection probability parameter value of $p=0$, the average values $p_{0}^{500}=p_{0}^{0}, p_{1}^{500}=p_{1}^{0}$, and $\Delta p_{0}^{500}=\Delta p_{1}^{500}=0$, that is, in a situation of lack of connections between agents the initial ratio of agents in each action remains unchanged.

2) As the network connection probability parameter value $p$ increases, both average $p_{0}^{500}$ and average $\Delta p_{0}^{500}$ decrease to a minimum value from where they increase (concave up), while average $p_{1}^{500}$ and average $\Delta p_{1}^{500}$ increase to a maximum from where they decrease (concave down).

This observation can be explained as follows: at time $t$, an agent $v_{i}$ randomly selects another agent $v_{j}(i \neq j)$. If $e_{i j}=1$, then the agent $v_{i}$ can obtain the opinion $o_{j}(t)$ of the agent $v_{j}$ by communicating with the agent $v_{j}$, and updates his/her opinion $o_{i}(t+1)$ based on the bounded confidence parameter value of $\varepsilon$; otherwise, $e_{i j}=0$, the agent $v_{i}$ can only notice the action $A_{j}(t)$ of the agent $v_{j}$ to update his/her opinion $o_{i}(t+1)$ based on the bounded confidence parameter value of $\varepsilon$. In this second case, it can happen as follows.

1) Agent $v_{i}$ with action nonsupport [i.e., $A_{i}(t)=0$ and $k=0$ ] selects an agent $v_{j}$ with action support [i.e., $A_{j}(t)=1$ and $\left.k=1\right]$, but cannot obtain the opinion $o_{j}(t)$ of agent $v_{j}$ at time $t$, then because the distance between the opinion value $o_{i}(t) \in[0,0.3)$ and the action value $A_{i}(t)=1$ is larger than the value of the bounded confidence parameter value, that is, $\left|1-o_{i}(t)\right|>\varepsilon=0.2$, $o_{i}(t) \in[0,0.3)$, situation $c$ of (2) implies that $o_{i}(t+1)=$ $o_{i}(t)$, which means that the opinion of agent $v_{i}$ at time $t$ cannot be influenced by the action $A_{j}(t)=1$ of the agent $v_{j}$

2) Agent $v_{i}$ with action support [i.e., $A_{i}(t)=1$ and $k=1$ ] selects an agent $v_{j}$ with action nonsupport [i.e., $A_{j}(t)=0$ and $k=0$ ], but cannot obtain the opinion $o_{j}(t)$ of the agent $v_{j}$ at time $t$, then because the distance between the opinion value $o_{i}(t) \in[0.3,1]$ and the action value $A_{j}(t)=0$ is larger than the bounded confidence parameter value $\varepsilon=0.2$, that is, $\left|0-o_{i}(t)\right|>\varepsilon=0.2$, 


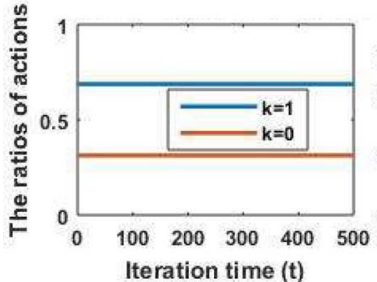

(a)

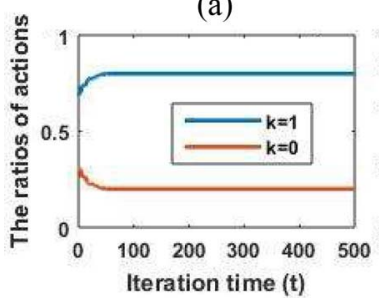

(c)

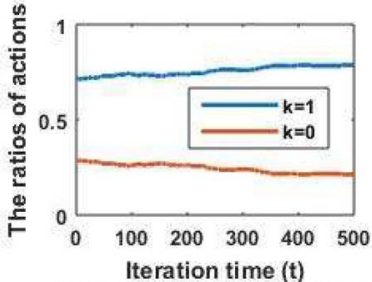

(b)

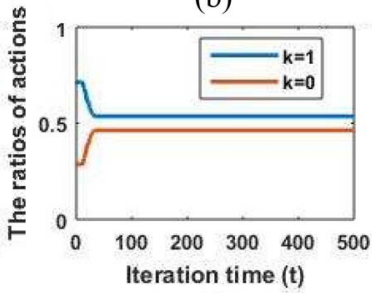

(d)
Fig. 4. Evolution of opinions and actions with different network connection probability parameter values $p(p=0,0.05,0.5,1)$ over time, with fixed parameter values $n=300, \varepsilon=0.2, h=0.3$, and $\alpha=0.5$. (a) Actions evolutions $(p=0)$. (b) Actions evolutions $(p=0.05)$. (c) Actions evolutions $(p=0.5)$. (d) Actions evolutions $(p=1)$.

$o_{i}(t) \in[0.3,1]$, situation $c$ in (2) implies that $o_{i}(t+1)=$ $o_{i}(t)$, which means that the opinion of agent $v_{i}$ at time $t$ cannot be influenced by the action $A_{j}(t)=0$ of the agent $v_{j}$.

Notice that because the initial opinions of agents $O(0)=$ $\left\{o_{1}(0), o_{2}(0), \ldots, o_{n}(0)\right\}$ are uniformly and randomly selected from $[0,1]$, and the opinion threshold of action choice parameter value is $h=0.3$, the opinions distribution width of agents with action nonsupport $(k=0)$ is smaller than the opinions' distribution width of agents with action support $(k=1)$, and the number of agents with action nonsupport $(k=0)$ is smaller than the number of agents with action support $(k=1)$ at time $t=0$, that is, $h<1-h$ and $N_{0}^{0}<N_{1}^{0}$. Therefore, in the process of opinions and actions evolutions, the probability that an agent selects another agent with action support $(k=1)$ is greater than the probability that an agent selects another agent with action nonsupport $(k=0)$.

The network connection probability among agents is divided into two situations, that is, $p=0$ and $0<p \leq 1$.

1) For network connection probability parameter value of $p=0$, the conditions for the actions of agents to remain unchanged (Proposition 3) are verified in this case as shown in Fig. 4(a).

2) For a positive network connection probability parameter value $(0<p \leq 1)$, there are four cases for the opinions and actions update: case 1: agents have different actions and connection edges: opinions and actions are updated based on other agents' opinions and bounded confidence, that is, situations $a$ and $b$ of (2) apply; case 2: agents have the same actions and connection edges: opinions and actions are updated based on other agents' opinions and bounded confidence, that is, situations $a$ and $b$ of (2) apply; case 3: agents have different actions but no connection edges: opinions and actions are updated based on other agents' actions and bounded confidence, that is, situations $c$ and $d$ of (2) apply; case 4: agents have the same actions but no connection edges: opinions

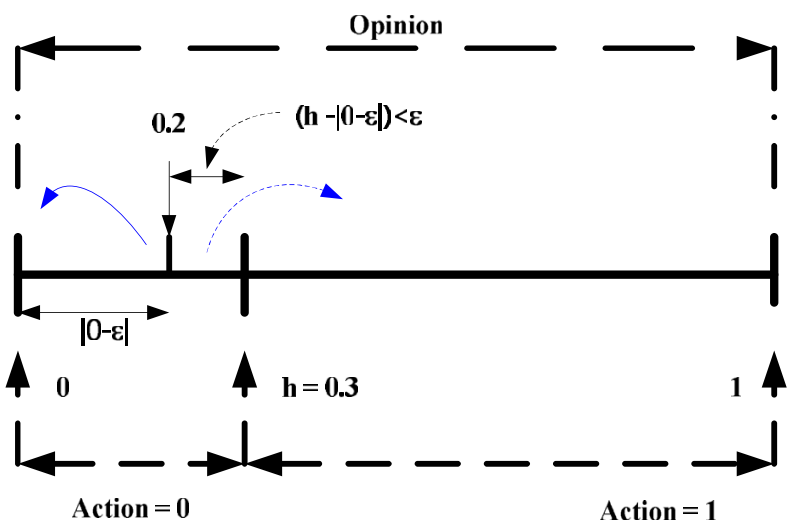

Fig. 5. Schematic of opinion and action evolution of agent in simulation experiments.

and actions are updated based on other agents' actions and bounded confidence, that is, situations $c$ and $d$ of (2) apply.

The final ratio $p_{k}^{500}$ and the total change ratio $\Delta p_{k}^{500}(k=$ $0,1)$ are influenced by cases 1,2 , and 4 rather than case 3 .

1) When the network connection probability parameter value is small [illustrated in Fig. 4(b) with $p=0.05$ ], there are few connections between agents in the social network. In this context, case 4 plays a dominant role in the evolution of opinions. Because the opinions update is based on the value of the noticed actions $(k=0,1)$, the opinions will come from both ends and become more extreme under bounded confidence, which relates to Propositions 1 and 2 of our theoretical study. Opinions of agents with action nonsupport $(k=0)$ belong to $[0, h)$, $|0-\varepsilon|=0.2<h=0.3$, and $(h-|0-\varepsilon|)<\varepsilon$; so in the process of the evolutions of opinions and actions, opinions of agents with action nonsupport $(k=0)$ are not completely attracted by the value of the discrete action nonsupport $(k=0)$, and some agents with action nonsupport $(k=0)$ at the initial time change their opinions and show action support $(k=1)$ at the final time (see Fig. 2). This leads to a decrease of the numbers of agents with nonsupport $(k=0)$ and to an increase of the number of agents with support $(k=1)$ at the final time. The schematic diagram of the evolutions of the opinions and actions evolutions is show in Fig. 5.

2) As the network connected probability parameter value $p$ increases, the number of communication edges increases, and the impact of the action nonsupport $(k=0)$ on the opinions of agents with action nonsupport $(k=0)$ decreases gradually, although it still plays a dominant role in the evolution of opinions. The opinions of agents with action support $(k=1)$ increase their impact on the opinions of the agents with action nonsupport $(k=0)$, which leads to more agents with action nonsupport $(k=0)$ at the initial time to change their opinions and show action support $(k=1)$ at the final time. So as the network connection probability parameter value $p$ increases from 0 , average $p_{0}^{500}$ and average $\Delta p_{0}^{500}$ values decrease until they reach their minimum values. 


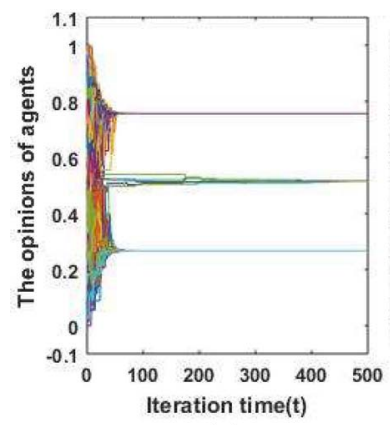

(a)

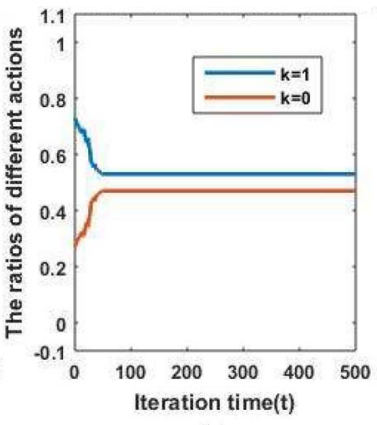

(b)
Fig. 6. Evolution of opinions and actions among $n=300$ agents over time, with fixed parameter values $p=1, \varepsilon=0.2, h=0.3$, and $\alpha=0.5$. (a) Opinions evolutions of agents. (b) Actions evolutions of agents.

3) As the network connection probability parameter value $p$ continues to increase from the value at which the aforementioned minimums are reached, the impact of the value of the action nonsupport $(k=0)$ on the opinions of agents with action nonsupport $(k=0)$ becomes weaker because the number of communication edges among agents with action nonsupport $(k=0)$ increases. The attraction of the opinions among the agents with action nonsupport $(k=0)$ is gradually smaller than the attraction of the opinions of the agents with action support $(k=1)$ on the opinions of the agents with action nonsupport $(k=0)$. Therefore, average $p_{0}^{500}$ and average $\Delta p_{0}^{500}$ values increase gradually, but average $p_{1}^{500}$ and average $\Delta p_{1}^{500}$ values decrease gradually. The evolutions of actions with $p=0.5$ and $p=1$ are shown in Fig. 4(c) and (d), respectively. Notice that for the specific case of being $p=1$, the ratio of the agents with action nonsupport $(k=0)$ at the final time is larger than at the initial time, which shows the number of agents with action nonsupport $(k=0)$ does not decrease but increases. The evolution of opinions and actions over time among agents with $p=1$ is shown in Fig. 6.

Therefore, as $p$ increases (with fixed $\varepsilon=0.2, h=0.3$, $\alpha=0.5$ ), after 500 independent realizations of the simulation, average $p_{0}^{500}$ and average $\Delta p_{0}^{500}$ values are concave up; while average $p_{1}^{500}$ and average $\Delta p_{1}^{500}$ values are concave down.

The number of agents in the social network has no obvious influence on the evolution of opinions and actions.

Notably, when bounded confidence changes, as the network connection probability parameter value increases, the average values of the final ratios and the total change ratios of agents with different actions may also change. We verified that when $0.1<\varepsilon<0.25$, as $p$ increases, average $p_{0}^{500}$ and average $\Delta p_{0}^{500}$ still are concave up, while average $p_{1}^{500}$ and average $\Delta p_{1}^{500}$ still are concave down.

\section{B. Impact of the Bounded Confidence and Opinion Threshold of Action Choice Parameters on the Evolution of Opinions and Actions}

In the following, we report on the impact of bounded confidence and opinion threshold of action choice parameters on the
TABLE III

Parameters Setting in Simulation Experiments in Section IV-B

\begin{tabular}{ll}
\hline \hline The numbers of agents & $n=500$ \\
Network connection probabilities & $p=0.1$ \\
Bounded confidence & $\varepsilon \in[0.1,0.5]$ \\
Opinion threshold of action choice & $h \in[0.1,0.9]$ \\
The convergence parameter & $\alpha=0.5$ \\
Iteration times & $t=0,1,2, \ldots, 500$ \\
\hline \hline
\end{tabular}

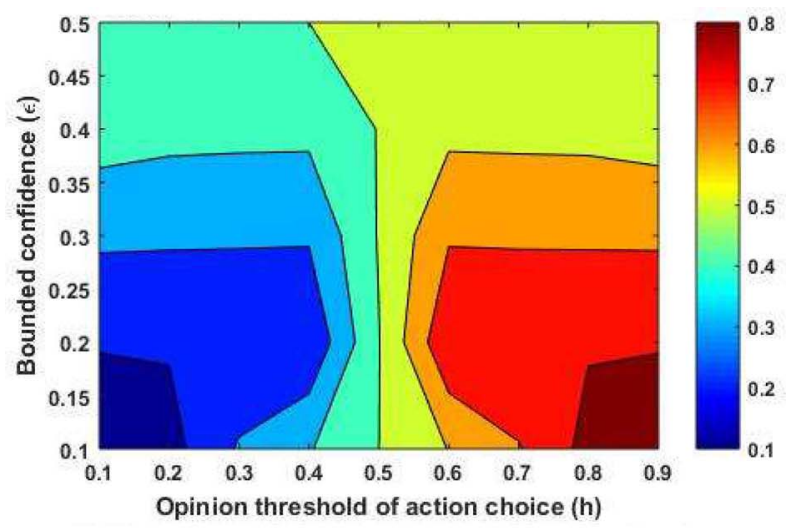

(a)

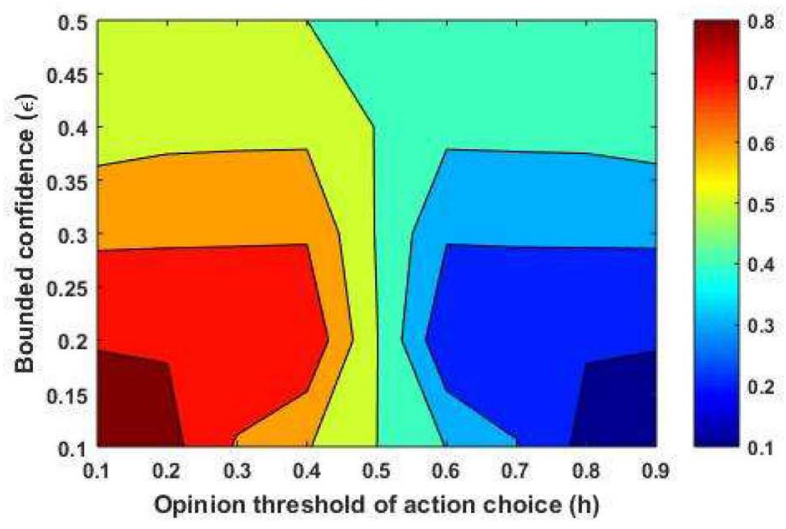

(b)

Fig. 7. Average final ratio of agents with different actions $(k=0$ or $k=1$ ), after 500 times independent simulation realizations, based on different bounded confidence and opinion threshold of action choice parameters, with fixed parameters $p=0.1, n=500, \alpha=0.5$, and $t=500$. (a) The average final ratio of agents with action $k=0$. (b) The average final ratio of agents with action $k=1$.

evolution of opinions and actions by in simulation experiments with parameters setting as per Table III.

Using the simulation method, we obtain the average final ratio of agents and the average total change ratio of agents with different action values in Figs. 7 and 9, respectively.

1) Average Final Ratio of Agents With Different Actions Under Different Bounded Confidence and Opinion Threshold of Action Choice Parameters: Fig. 7 shows the average final ratios of agents with different actions [Fig. 7(a) for action $k=0$; Fig. 7(b) for action $k=1$ ] with respect to the bounded confidence and opinion threshold of action choice parameters.

From Fig. 7, two observations are noticed regarding the effect of opinion threshold of action choice parameter and the bounded confidences parameter, respectively. 
1) As the opinion threshold of action choice parameter $h$ increases, average $p_{0}^{500}$ increases while average $p_{1}^{500}$ decreases, that is, the agents move from risk-preference to risk-conservative, as a result of the number of agents with action nonsupport $(k=0)$ increases while the number of agents with action support $(k=1)$ decreases. This observation has the following explanation: as the opinion threshold of action choice value increases, the initial ratio of agents with nonsupport action $(k=0)$ also increases but the initial ratio of agents with support action $(k=1)$ decreases, which leads to the increase of the final ratio of agents with nonsupport action $(k=0)$ and the decrease of the final ratio of agents with support action $(k=1)$.

2) As the bounded confidences parameter $\varepsilon$ increases: a) when the opinion threshold of action choice parameter verifies $h<0.28$, average $p_{0}^{500}$ increases while average $p_{1}^{500}$ decreases and $\mathrm{b}$ ) when the opinion threshold of action choice parameter verifies $0.72<h$, average $p_{0}^{500}$ decreases while average $p_{1}^{500}$ increases.

This observation shows that when the value of the opinion threshold of action choice is small, as the bounded confidence parameter increases, at the final time the number of agents with action nonsupport $(k=0)$ gradually increases, while the number of agents with action support $(k=1)$ gradually decreases; with opposite monotonicity trend when the value of the opinion threshold of the action choice value is high. This observation has the following explanation: in the simulation experiments, the network connection probability value $p=0.1$ means that the connection edges among the agents are sparse. Agent updates their opinions predominantly based on other agents' noticed actions, that is, situations $c$ and $d$ of (2), rather than on other agents' opinions. Therefore, the opinions of agents are strongly influenced by the value of the discrete action under bounded confidence. In the evolution process of opinions and actions, when $h<0.28$, as the bounded confidence parameter increases, more agents' opinions are attracted by the value of the action of nonsupport $(k=0)$ of agents, so the average final ratio of agents with action nonsupport $(k=0)$ increases while the average final ratio of agents with action support $(k=1)$ decreases.

The evolution of the two actions ( $k=0$ and $k=1$ ) over time with respect to the bounded confidence and opinion threshold of action choice parameters are depicted in Fig. 8. In Fig. 8(a1)-(a5) and (b1)-(b5), with opinion threshold of action choice parameter values $h=0.1$ and $h=0.2$, respectively, as the bounded confidence parameter increases, the number of agents with action nonsupport $(k=0)$ at the final time gradually increases, while the number of agents with action support $(k=1)$ at the final time gradually decreases; In Fig. 8(c1)-(c5) and (d1)-(d5), with $h=0.3$ and $h=0.4$, respectively, this monotonicity trend does not hold.

Due to the opposition characteristic between action nonsupport $(k=0)$ and action support $(k=1)$, when the opinion threshold of action choice verifies $0.72<h$, the increase of the bounded confidence value implies that more agents' opinions are attracted by the value of the action of support $(k=1)$ of agents, so the average final ratio of
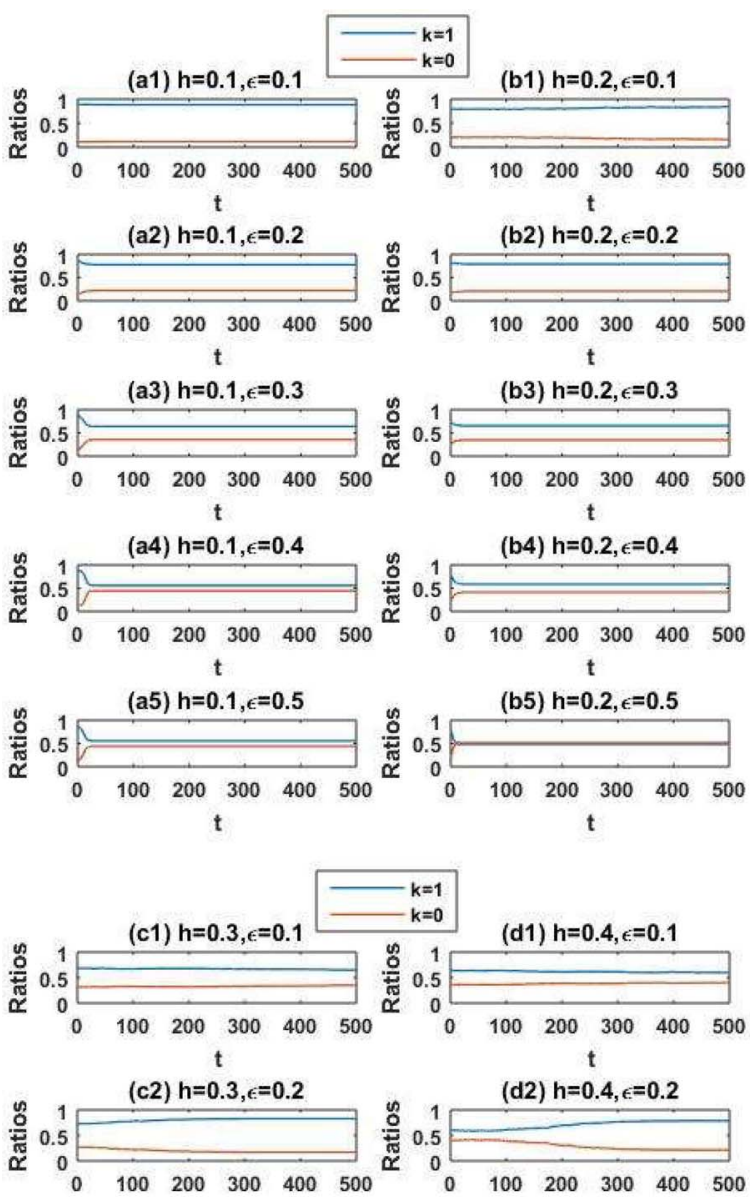

$\mathrm{t}$
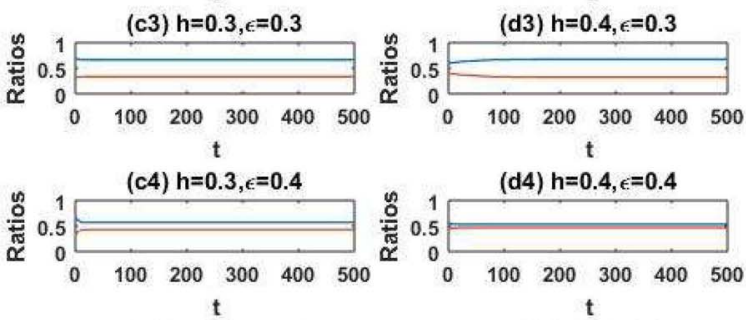

(c5) $\mathrm{h}=0.3, \epsilon=0.5$
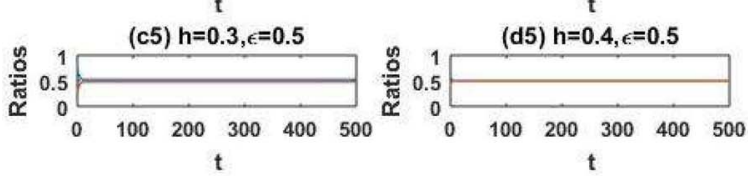

Fig. 8. Evolution of the two actions $(k=0$ or $k=1)$ over time with respect to different bounded confidence $\varepsilon$ and opinion threshold of action choice parameters $h$, with fixed parameter values $p=0.1, n=500$, and $\alpha=0.5$.

agents with action nonsupport $(k=0)$ decreases while the average final ratio of agents with action support $(k=1)$ increases.

2) Average Total Change Ratio of Agents With Different Actions Under Different Bounded Confidence and Opinion Threshold of Action Choice Parameters: Fig. 9 shows the average total change ratio of agents with different actions [Fig. 9(a) for action $k=0$; Fig. 9(b) for action $k=1$ ] with respect to the bounded confidence and opinion threshold of action choice parameters.

As before, two observations are drawn from Fig. 9. 


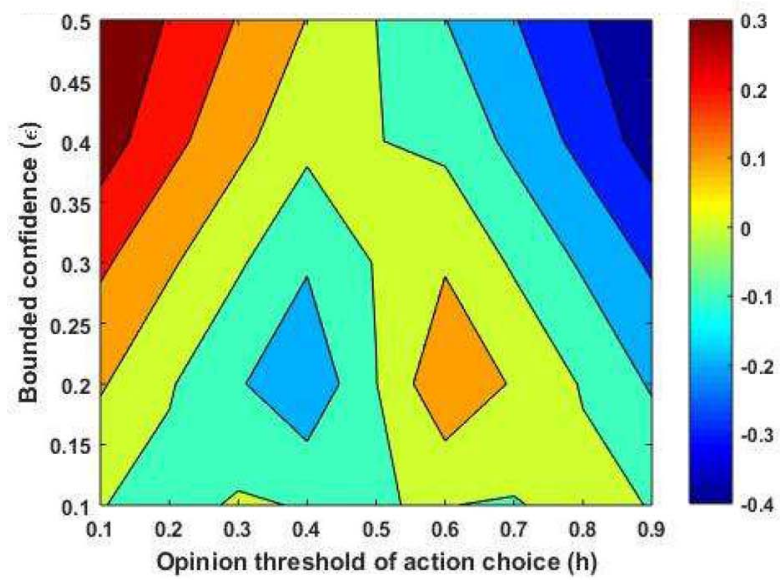

(a)

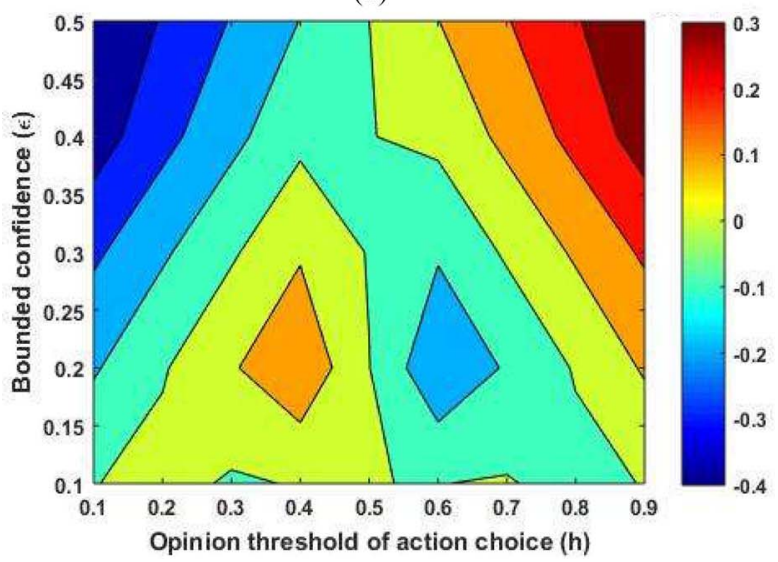

(b)

Fig. 9. Average total change ratio of agents with different actions $(k=0$ or $k=1$ ), after 500 times independent simulation realizations, based on different bounded confidence and opinion threshold of action choice values, with fixed parameters $p=0.1, n=500, \alpha=0.5$, and $t=500$. (a) The average total change ratio of agents with action $k=0$. (b) The average total change ratio of agents with action $k=1$.

1) As the opinion threshold of action choice parameter $h$ increases, when $0.38<\varepsilon<0.5$, average $\Delta p_{0}^{500}$ decreases while average $\Delta p_{1}^{500}$ increases. Thus, when bounded confidence parameter is high, as the opinion threshold of action choice parameter increases the total change ratio of agents with action nonsupport $(k=0)$ decreases while the total change ratio of agents with action support $(k=1)$ increases. The explanation of this observation is the following: first, as the opinion threshold of action choice parameter increases, at the initial time the ratio of agents with action nonsupport $(k=0)$ increases while the ratio of agents with action support $(k=1)$ decreases. Because the network connection probability parameter is a small value $(0.1)$, the update of agents' opinions based on other agents' noticed actions (i.e., situations $c$ and $d$ ) plays a dominant role in the evolution of opinions rather than the update of opinions based on other agents' opinions. In addition, larger opinions distribution width of agents with a certain action means a higher chance of that action to be chosen. Second, when the bounded confidence parameter is high and the value of the opinion threshold of action choice parameter is low, the opinions of agents with action support $(k=1)$ at the initial time are gradually attracted by the value of the noticed action nonsupport $(k=0)$, so at the final time they show action nonsupport $(k=0)$. The smaller the opinion threshold of action choice parameter is, the more agents change their initial actions support $(k=1)$ to action nonsupport $(k=0)$ at the final time, so the value of the total change ratio of agents with action nonsupport $(k=0)$ is higher. Third, as the opinion threshold of action choice parameter increases, more agents show action nonsupport $(k=0)$ at the final time, while the total change ratio of agents with action nonsupport $(k=0)$ decreases, because of the decrease of the value $\varepsilon-h$. As the increase of the opinion threshold of action choice parameter continues, the opinions of agents with action support $(k=0)$ at the initial time are gradually attracted by the value of the noticed action support $(k=1)$. Therefore, the average total change ratios of agents with action support $(k=1)$ increases.

2) As the bounded confidences parameter $\varepsilon$ increases: a) when the opinion threshold of action choice parameter verifies $h<0.28$, average $\Delta p_{0}^{500}$ increases while average $\Delta p_{1}^{500}$ decreases and b) when the opinion threshold of action choice parameter verifies $0.72<h$, average $\Delta p_{0}^{500}$ decreases while average $\Delta p_{1}^{500}$ increases.

This observation shows that when the opinion threshold of action choice parameter is low, the characteristic of agents is risk-preference; moreover, the larger the bounded confidence parameter is the larger the average total change ratio of the agent with action nonsupport is. However, when the opinion threshold of action choice parameter is high, the characteristic of agents is risk conservative; furthermore, the larger the bounded confidence value parameter is the larger average the total change ratio of the agent with action support is. This is explained because when the opinion threshold of action choice parameter is low, as the bounded confidence parameter increases the amount of opinions of agents with action support $(k=1)$ that are attracted by the value of agents' action $(k=0)$ increases, which means that more agents with action support $(k=1)$ at the initial time change their initial action to action nonsupport $(k=0)$ at the final time. The effect is opposite when the opinion threshold of action choice parameter is high and the bounded confidence parameter increases.

Therefore, in a context of a network connection probability parameter of $p=0.1$, number of agents $n=500$, convergence parameter value $\alpha=0.5$, iteration time $t=500$, and 500 independent realizations of the simulation, the impact of the bounded confidence and opinion threshold of action choice parameters on the evolution of opinions and actions evolutions are as follows.

1) As the opinion threshold of action choice parameter increases, the characteristic of agents changes from riskpreference to risk-conservative due to the increase of the number of agents with action nonsupport $(k=0)$ and the decrease of the number of agents with action support $(k=1)$.

2) When the opinion threshold of action choice parameter is low, increasing the bounded confidence parameter 
increases the number of agents with action nonsupport $(k=0)$ and decreases the number of agents with action support $(k=1)$ at the final time. However, when the value of the opinion threshold of the action choice parameter is high, increasing the bounded confidence parameter has the opposite effect at the final time.

3) When the bounded confidence parameter is high, increasing the opinion threshold of the action choice parameter decreases the total change ratio of agents with action nonsupport $(k=0)$ and increases the total change ratio of agents with action support $(k=1)$.

4) When the opinion threshold of action choice parameter is low, the characteristic of agents is risk preference, with the average total change ratio of the agent with action nonsupport being larger, the larger the bounded confidence parameter is. When the opinion threshold of the action choice parameter is high, the characteristic of agents is risk conservative, with the average total change ratio of the agent with action support being larger, larger the bounded confidence parameter.

\section{CONCLUSION}

This article proposed a model for opinions and actions evolution in social networks under bounded confidence. Both theoretical and empirical research results regarding the evolution of opinions and actions in social networks have been presented. The theoretical research results prove that the following.

1) Discrete actions can attract agents who trust the discrete action, and make the agents express extreme opinions, because:

a) if an agent always trusts the noticed discrete action of nonsupport to update his/her opinion, then the opinion of the agent can be completely attracted by the discrete action of nonsupport, until the opinion value becomes zero and keeps stable (Proposition 1);

b) if an agent always trusts the noticed discrete action of support to update his/her opinion, then the opinion of the agent can be completely attracted by the discrete action of support, until the opinion value equals one and keeps stable (Proposition 2).

2) When the network connection probability is 0 , due to the limitation of the bounded confidence, the actions of agents are unchanged (Proposition 3).

Simulation experimental results showed that social network connection probability, bounded confidence, and the opinion threshold of action choice parameters have strong impacts on the evolution of opinions and actions. However, the number of agents in the social network has no obvious influence on the evolution of opinions and actions.

The results in this article suggested that firms and organization should consider adding the social connections among the agents to decrease the appearance of extreme opinions. Although the results in this article are helpful to understand the evolution of opinions and actions in social networks, it is still a challenging task to collect real data to develop real data-driven SNOAEs.

\section{APPENDIX}

\section{A. Proof of Proposition 1}

Because $e_{i j}=0, A_{j}(t)=0$ and $\left|o_{i}(t)-A_{j}(t)\right| \leq \varepsilon$ at each time $t$, then based on situation $\mathrm{d}$ in (2) we can obtain $o_{i}(t+$ $1)=o_{i}(t)+\alpha\left(A_{j}(t)-o_{i}(t)\right)=o_{i}(t)+\alpha\left(0-o_{i}(t)\right)=(1-$ $\alpha) o_{i}(t)=(1-\alpha)^{t+1} o_{i}(0)$. Because $\alpha \in(0,0.5]$ and $o_{i}(0) \in$ $[0,1]$, obviously, $\lim _{t \rightarrow \infty} o_{i}(t)=0$.

\section{B. Proof of Proposition 2}

Because $e_{i j}=0, A_{j}(t)=1$, and $\left|o_{i}(t)-A_{j}(t)\right| \leq \varepsilon$ at each time $t$, then based on situation $d$ in (2) we can obtain $o_{i}(t+$ $1)=o_{i}(t)+\alpha\left(A_{j}(t)-o_{i}(t)\right)=o_{i}(t)+\alpha\left(1-o_{i}(t)\right)$. Because $\alpha \in(0,0.5]$, and $o_{i}(0) \in[0,1]$, then:

1) if $o_{i}(t) \in[0,1), o_{i}(t+1)-o_{i}(t)=\alpha\left(1-o_{i}(t)\right)>0$;

2) if $o_{i}(t)=1$, then $o_{i}(t+1)=o_{i}(t)=1$.

The sequence $\left\{o_{i}(t+1)\right\}$ is monotonic increasing and bounded above by 1 . Therefore, the sequence $\left\{o_{i}(t+\right.$ 1)\} converges and its limit is the upper bound, that is, $\lim _{t \rightarrow \infty} o_{i}(t)=1$.

\section{Proof of Proposition 3}

For a certain issue, let $o_{i}(t)$ and $o_{j}(t)$ be the opinions of agent $v_{i}$ and agent $v_{j}$ at time $t$, respectively; and let $A_{i}(t)$ and $A_{j}(t)$ be the actions of agent $v_{i}$ and agent $v_{j}$ at time $t$, respectively, where $o_{i}(t), o_{j}(t) \in[0,1], A_{i}(t), A_{j}(t) \in\{0,1\}$, $i, j=1,2, . ., n$ and $i \neq j$.

When network connection probability $p=0$, then for any pair of agents $v_{i}$ and $v_{j}$ we have $e_{i j}=0(i \neq j)$. Thus, an agent $v_{i}$ cannot obtain the opinion of the other agents but notice their actions, which are used to update his/her opinion based on bounded confidence $\varepsilon$. Therefore, in the process of opinion evolution situations $c$ and $d$ in (2) apply.

Case A: For an agent $v_{i}$ with opinion $o_{i}(t) \in[0, h)$, (1) implies that action $A_{i}(t)=0$ at time $t$. Let the noticed action $A_{j}(t)$ of other agent $v_{j}$ being used to update his/her opinion at time $t+1$. Then, we have the following.

Case A-1: If $A_{j}(t)=0$ and $\left|o_{i}(t)-A_{j}(t)\right|=o_{i}(t)>\varepsilon$, then according to situation $c$ of (2), it is: $o_{i}(t+1)=o_{i}(t) \in[0, h)$, $A_{i}(t+1)=0$.

Case A-2: If $A_{j}(t)=0,\left|o_{i}(t)-A_{j}(t)\right|=o_{i}(t) \leq \varepsilon$, then according to situation $d$ of (2), it is: $o_{i}(t+1)=o_{i}(t)+\alpha\left(A_{j}(t)-\right.$ $\left.o_{i}(t)\right)=(1-\alpha) o_{i}(t)$. Because $\alpha \in(0,0.5]$ and $o_{i}(t) \in[0, h)$, so $o_{i}(t+1)=(1-\alpha) o_{i}(t) \in[0, h), A_{i}(t+1)=0$.

Case A-3: If $A_{j}(t)=1$, because $o_{i}(t) \in[0, h)$ and $\varepsilon<$ $1-h$, then $\left|o_{i}(t)-A_{j}(t)\right|=1-o_{i}(t)>1-h>\varepsilon$. Therefore, according to situation $c$ of (2), it is: $o_{i}(t+1)=o_{i}(t)$ and $A_{i}(t+1)=0$.

Case B: For an agent $v_{i}$ with opinion $o_{i}(t) \in[h, 1]$, (1) implies that action $A_{i}(t)=1$ at time $t$. Let the noticed action $A_{j}(t)$ of other agent $v_{j}$ being used to update his/her opinion at time $t+1$. Then, we have the following.

Case B-1: If $A_{j}(t)=0$, because $o_{i}(t) \in[h, 1]$ and $\varepsilon<h$, then $\left|o_{i}(t)-A_{j}(t)\right|=o_{i}(t) \geq h>\varepsilon$. Therefore, according to situation $c$ of (2), it is: $o_{i}(t+1)=o_{i}(t)$ and $A_{i}(t+1)=1$.

Case B-2: If $A_{j}(t)=1$ and $\left|o_{i}(t)-A_{j}(t)\right|=1-o_{i}(t) \leq \varepsilon$, then according to situation $d$ of (2), it is: $o_{i}(t+1)=o_{i}(t)+$ $\alpha\left(A_{j}(t)-o_{i}(t)\right)=o_{i}(t)+\alpha\left(1-o_{i}(t)\right)$. Because $\alpha \in(0,0.5]$ and 
$o_{i}(t) \in[h, 1]$, so $\alpha\left(1-o_{i}(t)\right) \geq 0$. Therefore, $o_{i}(t+1) \geq o_{i}(t)$ and $A_{i}(t+1)=1$.

Case B-3: If $A_{j}(t)=1$ and $\left|o_{i}(t)-A_{j}(t)\right|=1-o_{i}(t)>\varepsilon$, then according to situation $c$ of (2), it is: $o_{i}(t+1)=o_{i}(t)$ and $A_{i}(t+1)=1$.

Summarizing, when $p=0, \varepsilon<h$ and $\varepsilon<1-h$, then $A_{i}(t+1)=A_{i}(t)(i=1,2, \ldots, n, t=0,1,2, \ldots)$.

\section{Proof of Proposition 4}

The interaction between agents in social network is divided into four cases.

Case 1: For any agent $v_{i} \in V^{0}(t)\left(o_{i}(t)=[0, h), A_{i}(t)=\right.$ $0)$, he/she randomly choose an agent $v_{p} \in V^{0}(t)\left(o_{p}(t)=\right.$ $\left.[0, h), A_{p}(t)=0\right)$ at time $t$, then $A_{i}(t+1)=A_{i}(t)=0$ no matter $e_{i p}=0$ or $e_{i p}=1$ in social network $G(V, E)$.

Case 2: For any agent $v_{i} \in V^{0}(t)\left(o_{i}(t)=[0, h)\right.$, $\left.A_{i}(t)=0\right)$, he/she randomly choose an agent $v_{j} \in V^{1}(t)$ $\left(o_{j}(t)=[h, 1], A_{j}(t)=1\right)$ at time $t$, because $e_{i j}=0$ and $\left|o_{i}(t)-A_{j}(t)\right|>\varepsilon$, then $A_{i}(t+1)=A_{i}(t)=0$.

Case 3: For any agent $v_{j} \in V^{1}(t)\left(o_{j}(t)=[h, 1], A_{j}(t)=1\right)$, he/she randomly choose an agent $v_{i} \in V^{0}(t)\left(o_{i}(t)=[0, h)\right.$, $\left.A_{i}(t)=0\right)$ at time $t$, because $e_{j i}=0$ and $\left|o_{j}(t)-A_{i}(t)\right|>\varepsilon$, then $A_{i}(t+1)=A_{i}(t)=1$.

Case 4: For any agent $v_{j} \in V^{1}(t)\left(o_{j}(t)=[h, 1], A_{j}(t)=1\right)$, he/she randomly choose an agent $v_{p} \in V^{1}(t)\left(o_{p}(t)=[h, 1]\right.$, $\left.A_{p}(t)=1\right)$ at time $t$, then $A_{i}(t+1)=A_{i}(t)=1$ no matter $e_{i p}=0$ or $e_{i p}=1$ in social network $G(V, E)$.

Therefore, in social network $G(V, E)$, for any two agents $v_{i} \in V^{0}(t)$ and $v_{j} \in V^{1}(t)$ at time $t$, if $e_{i j}=0$ and $\left|o_{i}(t)-A_{j}(t)\right|>\varepsilon$, and $e_{j i}=0$ and $\left|o_{j}(t)-A_{i}(t)\right|>\varepsilon$, then $A_{i}(t+1)=A_{i}(t)$ and $A_{j}(t+1)=A_{j}(t)$.

\section{REFERENCES}

[1] P. F. Anderson, "Marketing, strategic planning and the theory of the firm," J. Market., vol. 46, no. 2, pp. 15-26, Apr. 1982.

[2] R. A. Barrio, T. Govezensky, R. Dunbar, G. Iñiguez, and K. Kaski, "Dynamics of deceptive interactions in social networks," J. Roy. Soc. Interface, vol. 12, no. 112, pp. 463-474, 2015.

[3] R. L. Berger, "A necessary and sufficient condition for reaching a consensus using DeGroot's method,' J. Amer. Stat. Assoc., vol. 76, no. 374, pp. 415-418, 1981.

[4] R. Black, "Continuous opinion dynamics under bounded confidence: A survey," Int. J. Mod. Phys. C, vol. 18, no. 12, pp. 1819-1838, 2007.

[5] S. Boccaletti, V. Latora, Y. Moreno, M. Chavez, and D. U. Hwang, "Complex networks: Structure and dynamics," Phys. Rep., vol. 424, nos. 4-5, pp. 175-308, 2006.

[6] C. Castellano, S. Fortunato, and V. Loreto, "Statistical physics of social dynamics," Rev. Mod. Phys., vol. 81, no. 2, pp. 591-646, 2009.

[7] J. Castro, J. Lu, G. Zhang, Y. C. Dong, and L. Martínez, "Opinion dynamics-based group recommender systems," IEEE Trans. Syst., Man, Cybern., Syst., vol. 48, no. 12, pp. 2394-2406, Dec. 2018.

[8] M. Chandrashekaran, B. A. Walker, J. C. Ward, and P. H. Reingen, "Modeling individual preference evolution and choice in a dynamic group setting," J. Market. Res., vol. 33, no. 2, pp. 211-223, 1996.

[9] G. Deffuant, D. Neau, and F. Amblard, "Mixing beliefs among interacting agents," Adv. Complex Syst., vol. 3, nos. 1-4, pp. 87-98, 2000.

[10] M. H. DeGroot, "Reaching a consensus," J. Amer. Stat. Assoc., vol. 69, no. 345, pp. 118-121, 1974.

[11] Z. G. Ding, X. Chen, Y. C. Dong, and F. Herrera, "Consensus reaching in social network DeGroot model: The roles of the self-confidence and node degree," Inf. Sci., vol. 486, pp. 62-72, Jun. 2019.

[12] Y. C. Dong, Z. G. Ding, L. Martínez, and F. Herrera, "Managing consensus based on leadership in opinion dynamics," Inf. Sci., vols. 397-398, pp. 187-205, Aug. 2017.
[13] Y. C. Dong, Q. B. Zha, H. J. Zhang, and F. Herrera, "Consensus reaching and strategic manipulation in group decision making with trust relationships," IEEE Trans. Syst., Man, Cybern., Syst., early access, Jan. 13, 2020, doi: 10.1109/TSMC.2019.2961752.

[14] Y. C. Dong et al., "Consensus reaching in social network group decision making: Research paradigms and challenges," Knowl. Based Syst., vol. 162, pp. 3-13, Dec. 2018.

[15] Y. C. Dong, M. Zhan, Z. G. Ding, H. M. Liang, and F. Herrera, "Numerical interval opinion dynamics with social network: Stable state and consensus," IEEE Trans. Fuzzy Syst., early access, Dec. 2, 2019, doi: 10.1109/TFUZZ.2019.2956907.

[16] Y. C. Dong, M. Zhan, G. Kou, Z. G. Ding, and H. M. Liang, "A survey on the fusion process in opinion dynamics," Inf. Fusion, vol. 43, pp. 57-65, Sep. 2018

[17] K. Fan and W. Pedrycz, "Opinion evolution influenced by informed agents," Physica A, Stat. Mech. Appl., vol. 462, pp. 431-441, Nov. 2016.

[18] L. Fotia, F. Messina, D. Rosaci, and G. M. L. Sarnè, "Using local trust for forming cohesive social structures in virtual communities," Comput. J., vol. 60, pp. 1717-1727, Nov. 2017.

[19] N. E. Friedkin and E. C. Johnsen, "Social influence and opinions," J. Math. Sociol., vol. 15, nos. 3-4, pp. 193-205, 1990.

[20] N. E. Friedkin and E. C. Johnsen, "Social influence networks and opinion change," in Advances in Group Processes, vol. 16. Greenwich, U.K. JAI Press, 1999, pp. 1-29.

[21] N. E. Friedkin, A. V. Proskurnikov, R. Tempo, and S. E. Parsegov, "Network science on belief system dynamics under logic constraints," Science, vol. 354, no. 6310, pp. 321-326, 2016.

[22] S. Galam, "Minority opinion spreading in random geometry," Eur. Phys. J. B, Condensed Matter Complex Syst., vol. 25, no. 4, pp. 403-406, 2002.

[23] R. Hegselmann, S. König, S. Kurz, C. Niemann, and J. Rambau, "Optimal opinion control: The campaign problem," J. Artif. Soc. Soc. Simul., vol. 18, no. 3, p. 47, 2014.

[24] R. Hegselmann and U. Krause, "Opinion dynamics and bounded confidence: Models, analysis and simulation,” J. Artif. Soc. Soc. Simul., vol. 5 , no. 3, pp. 3-33, 2002.

[25] R. A. Holley and T. M. Liggett, "Ergodic theorems for weakly interacting infinite systems and the voter model," Ann. Probab., vol. 3 no. 4, pp. 643-663, 1975.

[26] J. Lorenz, "Heterogeneous bounds of confidence: Meet, discuss and find consensus," Complexity, vol. 15, no. 4, pp. 43-52, 2010.

[27] N. Khalil, M. M. San, and R. Toral, "Zealots in the mean-field noisy voter model," Phys. Rev. E, vol. 97, no. 1, 2018, Art. no. 012310.

[28] P. Krapivsky and S. Redner, "Dynamics of majority rule in twostate interacting spin systems," Phys. Rev. Lett., vol. 90, Jun. 2003, Art. no. 238701.

[29] A. Leshem and A. Scaglione, "The impact of random actions on opinion dynamics," IEEE Trans. Signal Inf. Process. Over Netw., vol. 4, no. 3, pp. 576-584, Sep. 2018.

[30] Y. T. Liu, Y. C. Dong, H. M. Liang, F. Chiclana, and E. HerreraViedma, "Multiple attribute strategic weight manipulation with minimum cost in a group decision making context with interval attribute weights information," IEEE Trans. Syst., Man, Cybern., Syst., vol. 49, no. 10, pp. 1981-1992, Oct. 2019, doi: 10.1109/TSMC.2018.2874942.

[31] X. Luo, C. W. Jiang, W. P. Wang, Y. Xu, J.-H. Wang, and W. B. Zhao, "User behavior prediction in social networks using weighted extreme learning machine with distribution optimization," Future Gen. Comput. Syst., vol. 93, pp. 1023-1035, Apr. 2019.

[32] G.-X. Luo, Y. Liu, Q.-A. Zeng, S.-M. Diao, and F. Xiong, "A dynamic evolution model of human opinion as affected by advertising," Physica A, Stat. Mech. Appl., vol. 414, pp. 254-262, Nov. 2014.

[33] V. Mahajan, E. Muller, and F. M. Bass, "New product diffusion models in marketing: A review and directions for research," J. Market., vol. 54, no. 1, pp. 1-26, 1990.

[34] A. C. R. Martins, "Continuous opinions and discrete actions in opinion dynamics problems," Int. J. Mod. Phys. C, vol. 19, no. 04, pp. 617-624, 2008.

[35] N. Masuda, N. Gibert, and S. Redner, "Heterogeneous voter models," Phys. Rev. E, vol. 82, no. 1, 2010, Art. no. 010103.

[36] P. D. Meo, E. Ferrara, D. Rosaci, and G. M. L. Sarnè, "Trust and compactness in social network groups," IEEE Trans. Cybern., vol. 45, no. 2, pp. 205-216, Feb. 2015.

[37] E. Presutti and H. Spohn, "Hydrodynamics of the voter model," Ann. Probab., vol. 11, no. 4, pp. 867-875, 1983.

[38] S. Ríos, F. Aguilera, J. D. Nuñez-Gonzalez, and M. Graña, "Semantically enhanced network analysis for influencer identification in online social networks," Neurocomputing, vol. 326, pp. 71-81, Jan. 2019. 
[39] J. Semonsen, C. Griffin, A. Squicciarini, and S. Rajtmajer, "Opinion dynamics in the presence of increasing agreement pressure," IEEE Trans. Cybern., vol. 49, no. 4, pp. 1270-1278, Apr. 2019.

[40] X. Shi, J. D. Cao, G. H. Wen, and M. Perc, "Finite-time consensus of opinion dynamics and its applications to distributed optimization over digraph," IEEE Trans. Cybern., vol. 49, no. 10, pp. 3767-3779, Oct. 2019.

[41] A. Sîrbu, V. Loreto, V. D. P. Servedio, and F. Tria, "Opinion dynamics: Models, extensions and external effects," in Participatory Sensing, Opinions and Collective Awareness. Cham, Switzerland: Springer, May 2016, pp. 363-401.

[42] F. Slanina and H. Lavicka, "Analytical results for the Sznajd model of opinion formation," Eur. Phys. J. B, Condensed Matter Complex Syst., vol. 35, no. 2, pp. 279-288, 2003.

[43] X. Song, W. Shi, Y. Ma, and C. Yang, "Impact of informal networks on opinion dynamics in hierarchically formal organization," Physica A, Stat. Mech. Appl., vol. 436, pp. 916-924, Oct. 2015.

[44] V. Sood, T. Antal, and S. Redner, "Voter models on heterogeneous networks," Phys. Rev. E, vol. 77, no. 4, 2008, Art. no. 041121.

[45] K. Sznajd-Weron and J. Sznajd, "Opinion evolution in closed community," Int. J. Mod. Phys. C, vol. 11, no. 6, pp. 1157-1165, 2000.

[46] C. J. Tessone, R. Toral, P. Amengual, H. S. Wio, and M. San Miguel, "Neighborhood models of minority opinion spreading," Eur. Phys. J. B, Condensed Matter Complex Syst., vol. 39, no. 4, pp. 535-544, 2004.

[47] R. Urena, G. Kou, Y. C. Dong, F. Chiclana, and E. Herrera-Viedma, "A review on trust propagation and opinion dynamics in social networks and group decision making frameworks," Inf. Sci., vol. 478, pp. 461-475, Apr. 2019.

[48] G. Weisbuch, G. Deffuant, F. Amblard, and J. Nadal, "Meet, discuss and segregate," Complexity, vol. 7, no. 3, pp. 55-63, 2002.

[49] C. W. Wu, "Synchronization and convergence of linear dynamics in random directed networks," IEEE Trans. Autom. Control, vol. 51, no. 7, pp. 1207-1210, Jul. 2006.

[50] Y. Z. Wu, Y. C. Dong, J. D. Qin, and W. Pedrycz, "Flexible linguistic expressions and consensus reaching with accurate constraints in group decision-making," IEEE Trans. Cybern., vol. 50, no. 6, pp. 2488-2501, Jun. 2020, doi: 10.1109/tcyb.2019.2906318.

[51] Q. B. Zha, Y. C. Dong, H. J. Zhang, F. Chiclana, and E. Herrera-Viedma, "A personalized feedback mechanism based on bounded confidence learning to support consensus reaching in group decision making," IEEE Trans. Syst., Man, Cybern., Syst., early access, Oct. 28, 2019, doi: 10.1109/TSMC.2019.2945922.

[52] M. Zhan, H. M. Liang, Z. G. Ding, and Y. C. Dong, "Uncertain opinion evolution with bounded confidence effects in social networks," J. Syst. Sci. Syst. Eng., vol. 28, no. 4, pp. 494-509, 2019.

[53] M. Zhan, H. M. Liang, G. Kou, Y. C. Dong, and S. Yu, "Impact of social network structures on uncertain opinion formation," IEEE Trans. Comput. Soc. Syst., vol. 6, no. 4, pp. 670-679, Aug. 2019.

[54] Y. Y. Zhao and G. Kou, "Bounded confidence-based opinion formation for opinion leaders and opinion followers on social networks," Stud. Informat. Control, vol. 23, no. 2, pp. 153-162, 2014.

[55] Y. Y. Zhao, L. B. Zhang, M. F. Tang, and G. Kou, "Bounded confidence opinion dynamics with opinion leaders and environmental noises," Comput. Oper. Res., vol. 74, pp. 205-213, Oct. 2016.

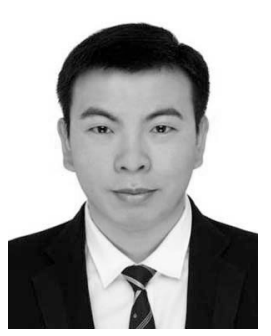

Min Zhan received the B.S. degree in environmental sciences and accounting from Northeast Forestry University, Harbin, China, and the M.S. degree in enterprise management from the Guangxi University of Science and Technology, Liuzhou, China, and the $\mathrm{Ph} . \mathrm{D}$. degree in management system engineering from Sichuan University, Chengdu, China in 2011, 2015, and 2019 respectively.

$\mathrm{He}$ is currently a Lecturer with the Institute of Big Data and Internet Innovation, Hunan University of Technology and Business, Changsha, China. He has published several international journal papers in Information Fusion, IEEE TRansactions on FuZzy Systems, IEEE TRANSACtions on Computational Social Systems, and Journal of Systems Science and Systems Engineering. His research interests include social network analysis and opinion dynamics.

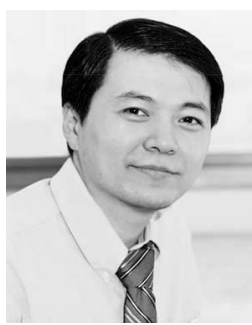

Gang Kou received the B.S. degree from the Department of Physics, Tsinghua University, Beijing, China, the master's degree from the Department of Computer Science, University of Nebraska at Omaha, Omaha, NE, USA, and the $\mathrm{Ph} . \mathrm{D}$. degree in information technology from the College of Information Science and Technology, University of Nebraska at Omaha in 1997, 2003, and 2006 respectively.

$\mathrm{He}$ is a Distinguished Professor with the Chang Jiang Scholars Program, Southwestern University of Finance and Economics, Chengdu, China. He was a Professor with the School of Management and Economics, University of Electronic Science and Technology of China, Chengdu, and a Research Scientist with Thomson R\&D Company, Moulineaux, France. He has published more than 100 papers in various peer-reviewed journals.

Dr. Kou is a Managing Editor of International Journal of Information Technology \& Decision Making and the Managing Editor-in-Chief of Financial Innovation (SSCI). He is also an Editor for the following journals, such as Decision Support Systems, European Journal of Operational Research, and Technological and Economic Development of Economy.

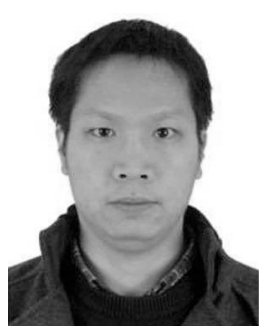

Yucheng Dong (Senior Member, IEEE) received the B.S. and M.S. degrees in mathematics from Chongqing University, Chongqing, China, in 2002 and 2004, respectively, and the Ph.D. degree in management from Xi'an Jiaotong University, Xi'an, China, in 2008

$\mathrm{He}$ is currently a Professor with the Business School, Sichuan University, Chengdu, China. He has authored or coauthored more than 100 international journal papers in Decision Support Systems. European Journal of Operational Research, IEEE Transactions, IISE Transactions, Omega, Risk Analysis, and Scientific Data. His current research interests include decision analysis, human dynamics, and big data analytics.

Prof. Dong has been identified by Clarivate as Highly Cited Researcher in the field of computer science. He is an Area Editor/Associate Editor of Computers and Industrial Engineering, Group Decision and Negotiation, the IEEE TRANSACTIONS ON Systems, MAN, AND CybERnETICS: Systems, and Information Fusion.

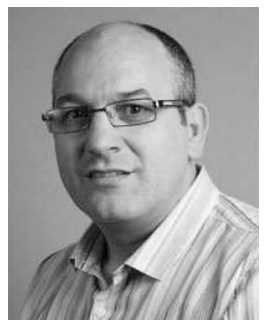

Francisco Chiclana (Senior Member, IEEE) received the B.Sc. and Ph.D. degrees in mathematics from the University of Granada, Granada, Spain, in 1989 and 2000, respectively.

$\mathrm{He}$ is a Professor of Computational Intelligence and Decision Making with the School of Computer Science and Informatics, Faculty of Computing, Engineering and Media, De Montfort University, Leicester, U.K. He has organized and chaired special sessions/workshops in many major international conferences in research areas as fuzzy preference modeling, decision support systems, consensus, recommender systems, social networks, rationality/consistency, and aggregation.

Prof. Chiclana is currently a Highly Cited Researcher in Computer Sciences (according to Essential Science Indicators by Clarivate Analytics). He is an Associate Editor and the Guest Editor for several ISI indexed journals.

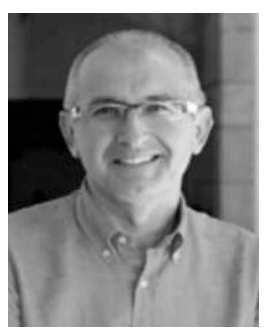

Enrique Herrera-Viedma (Senior Member, IEEE) received the M.Sc. and Ph.D. degrees in computer science from the University of Granada, Granada, Spain, in 1993 and 1996, respectively.

$\mathrm{He}$ is currently a Professor of Computer Science and Artificial Intelligence and the Vice-President of Research and Knowledge Transfer with the University of Granada. His H-index is 85 with more than 24000 citations received in Web of Science and 97 in Google Scholar with more than 37500 citations received. He has been identified as one of the world's most influential researchers by the Shanghai Center and Thomson Reuters/Clarivate Analytics in both Computer Science and Engineering from 2014 to 2020. His current research interests include group decision making, consensus models, linguistic modeling, aggregation of information, information retrieval, bibliometrics, digital libraries, Web quality evaluation, recommender systems, and social media.

Prof. Herrera-Viedma was the Vice President for Publications with the IEEE Systems, Man, and Cybernetics Society from 2019 to 2020 and an Associate Editor for several journals, such as the IEEE TRANSACTIONS ON FUZZY SYSTEMS, the IEEE TRANSACTIONS ON SYSTEMS, MAN AND CYBERNETICS: Systems, the IEEE TRANSACTIONS ON INTELLIGENT Transport Systems, Information Science, Applied Soft Computing, Soft Computing, Fuzzy Optimization and Decision Making, and KnowledgeBased Systems. 